\section{Cellular Physiology and Biochemistry}

Cell Physiol Biochem 2018;47:2380-2387

DOI: $10.1159 / 000491610$

Published online: July 09, 2018

Accepted: May 09, 2018

This article is licensed under the Creative Commons Attribution-NonCommercial-NoDerivatives 4.0 International License (CC BY-NC-ND) (http://www.karger.com/Services/OpenAccessLicense). Usage and distribution for commercial purposes as well as any distribution of modified material requires written permission.

Original Paper

\title{
The Effect of Mecp2 on Heart Failure
}

\author{
Chunli Wang Fei Wang Qing Cao Zhen Li Liya Huang Shuyan Chen \\ Department of Geriatrics, Xinhua Hospital Affiliated to Shanghai Jiao Tong University School of \\ Medicine, Shanghai, China
}

\section{Key Words}

Mecp2 $•$ Heart failure Protein-protein interactions - Functional enrichment

\begin{abstract}
Background/Aims: Heart failure is the end result of various kinds of cardiovascular diseases. It has a high rate of morbidity and mortality. This article aims to determine the effect of $\mathrm{MeCP} 2$, a key epigenetic regulator, on heart failure. Methods: The genes associated with heart failure were selected and analyzed using Gene Ontology (GO) term analysis and proteinprotein interaction (PPI) network analysis. Significantly up- or downregulated genes in a heart failure animal model were identified, and the genes that had the same or opposite alteration trends as MeCP2 were also recognized. Eighteen hub genes were picked based on topological parameters, and then aberrantly expressed genes with MeCP2 overexpression or knockout were analyzed by GO term, KEGG pathway and PPI analyses. Results: MeCP2 was downregulated in the heart failure animal model. Through comparison and alignment, 10 dysregulated genes were selected from the 18 hub genes (JAK1, SETD1B, HRC, TTN, LYZ2, TPM3, MYH11, MYH6, ALOX5AP, DECR1). These genes were mainly enriched in cytoskeletal regulation mediated by Rho GTPase and inflammation mediated by chemokine and cytokine signaling pathways. Conclusions: These dysregulated genes provide a better understanding of the underlying mechanisms of the effect of MeCP2 on heart failure and might be used as targets and prognostic markers of heart failure.
\end{abstract}

\section{Introduction}

(C) 2018 The Author(s) Published by S. Karger AG, Basel

$\mathrm{MeCP} 2$, a nuclear protein, is one member of a family of methyl-CpG-binding proteins. It consists of multiple structural domains, including a methyl-CpG-binding domain (MBD) and a transcriptional repression domain (TRD). The MBD binds to methylated DNA, and the TRD recruits HDAC-containing complexes via the $\mathrm{mSin} 3 \mathrm{~A}$ corepressor to repress transcription [1, 2] (Fig. 1). Recently, it was found that MeCP2 has some unmethylated and active regulatory regions [3]. MeCP2 was first identified as the cause of Rett syndrome (RTT) because of its X-linked gene mutation [4]. MeCP2 function in specific brain regions has been studied extensively, and many studies have confirmed that MeCP2 knockdown or overexpression results in the inverse dysregulation of thousands of genes [5-8]. Some researchers have

C. Wang and F. Wang contributed equally to this work. 
found that MeCP2 mRNA transcripts are highly expressed in lung, skeletal muscle and heart and moderately expressed in brain [9-12], whereas the protein is abundant in brain, lung and spleen; moderately abundant in kidney and heart; and hardly detectable in liver, stomach and small intestine [12]. MeCP2 is involved in the pathologies of many tissues and cells. It has received increasing attention as a nucleus-residing transcriptional regulator in various tissues and cells.

Epigenetic gene regulation plays a crucial role in cardiac fibrosis [13]. MeCP2, as an important epigenetic regulator, is considered to be a critical modulator. MeCP2 is increased in cardiac fibrosis tissue, and siRNA-MeCP2 reduces neonatal rat cardiac fibroblast cell viability by inhibiting cell proliferation [14]. In human and mouse heart failure, MeCP2 is repressed, and prevention of MeCP2 repression in mice after chronic cardiac pressure overload aggravates cardiac hypertrophy and mitochondrial and contractile dysfunction. In contrast, ablation of MeCP2 expression in cardiac myocytes in vivo facilitates functional recovery from cardiac failure by mechanical unloading of the heart [15]. It has also been reported that MeCP2-overexpressing human endothelial cells have impaired migration capacity and angiogenic activity, whereas MeCP2-silenced endothelial cells have improved endothelial migration and tube formation capacity [16]. All these data indicate the importance of MeCP2 as a regulator in cardiovascular diseases.

Based on the important role of MeCP2 in cardiovascular diseases, this article aims to further identify genes related to regulation of MeCP2 in heart failure. The original dataset (GSE52796) was downloaded from the Gene Expression Omnibus (GEO, http://www.ncbi. nlm.nih.gov/geo/). The data were extracted and handled with homogenization. Subsequently, the differentially expressed genes (DEGs) were screened, followed by enrichment analyses for Gene Ontology (GO) and pathway enrichment analysis. Protein-protein interaction (PPI) network analyses were done, and the candidate genes from the PPI network analyses were further analyzed.

\section{Materials and Methods}

\section{Initial datasets}

The initial gene expression profiles of GSE52786 were downloaded from the GEO database with the GPL6887 annotation platform. The dataset contained 49 samples, including wild-type (WT), MeCP2overexpressing (TG), and MeCP2-knockout (KO) mice; healthy mice (Sham) that underwent transverse aortic constriction (TAC); and mice that underwent removal of the aortic stenosis (reversible TAC, rTAC) [15].

\section{Process and tools of data analysis}

Two tools were used to screen DEGs for further alignment. One was GE02R, with an indicator-adjusted $\mathrm{P}$ value $<0.05$. The other was online Morpheus (https://software.broadinstitute.org/morpheu s/), using the indicator $\mathrm{P}$ value $<0.05$ and $|\log \mathrm{FC}|>1$. We performed enrichment analyses for GO terms using three tools for comparison. One was Cytoscape software. The plugin BiNGO was used to perform GO categories in Cytoscape. The predominant functional themes of a given gene set of a map were outputted. The second tool was online DAVID (https://david.ncifcrf.gov/). The third was online GoMiner (https://discover.nci. nih.gov/gominer/index.jsp). PPI information was evaluated by using the online database STRING (http:// www.string-db.org/). The DEGs were pasted into STRING, validated interactions were selected, and then the PPI network data were imported to Cytoscape. The plugin MCODE was used to screen the modules of PPI network in Cytoscape.

\section{Category and group of data}

The data were classified into two categories, the healthy group and the TAC group. The up- and downregulated genes were compared between the healthy and TAC groups. Through GO term and PPI analyses, the dysregulated genes under TAC were recognized. As another article reported [15], MeCP2 was downregulated under TAC and restored under rTAC. Next, we screened and aligned the genes with the same 

Cellular Physiology Cell Physiol Biochem 2018;47:2380-2387 \begin{tabular}{l|l} 
DOI: 10.1159/000491610 & $\begin{array}{l}\text { O 2018 The Author(s). Published by S. Karger AG, Basel } \\
\text { www.karger.com/cpb }\end{array}$ \\
\hline
\end{tabular}
Wang et al.: The Effect of MeCP2 on Heart Failure

regulation trend as MeCP2 and the genes with the reverse regulation trends from MeCP2 under TAC and rTAC. That is, if a gene was downregulated under TAC and upregulated under rTAC, it was aligned as the same trend as MeCP2; otherwise, it was identified as the opposite trend. We identified the pathways that were enriched among these genes. Last, through the analysis of MeCP2 overexpression and knockdown, we found key genes and pathways regulated by MeCP2.

\section{Results}

\section{Genes dysregulated under TAC}

We analyzed the up- and downregulated genes under TAC, finding 111 upregulated genes and 195 downregulated genes. GO term analysis through an online tool (http:// pantherdb.org/) showed that up- and downregulated genes were mainly enriched in biological regulation, cellular process, metabolic process and developmental process under the biological process category; in transporter activity, binding, catalytic activity and receptor activity under the molecular function category; and in cell junction, cell part, membrane and organelle under the cellular component category (Fig. 2). Panther pathways are shown in Fig. 3. The top density enrichment of all pathways was inflammation mediated by chemokine and cytokine signaling. These findings demonstrate that inflammatory reactions play an important role in heart failure. Some classical signal pathways were involved in TAC, including apoptosis signaling, PI3K signaling, Wnt signaling and the p53 pathway. PPI networks are shown in Fig. 4. The top hub genes were Myh6 (myosin heavy polypeptide 6 cardiac muscle), Myh11 (myosin heavy polypeptide 11 smooth muscle), Tpm3 (tropomyosin 3, gamma),

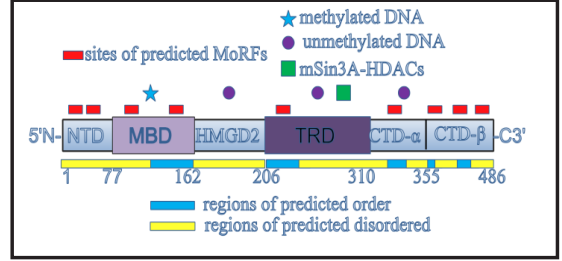

Fig. 1. Functional regions of MeCP2.

Fig. 2. Gene ontology analysis of differentially expressed genes under TAC. A. Upregulated genes by Panther molecular function category. B. Upregulated genes by Panther biological process category. C. Upregulated genes by Panther cellular component category. D Downregulated genes

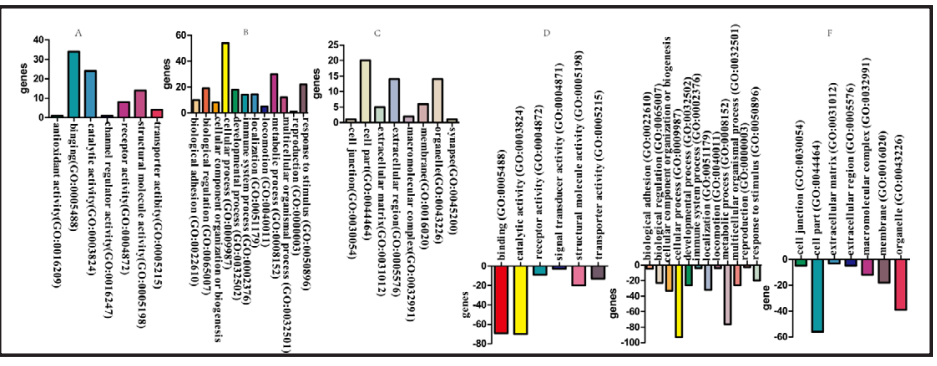
by Panther molecular function category. E. Downregulated genes by Panther biological process category. F. Downregulated genes by Panther cellular component category.

Fig. 3. Pathway analysis of upand downregulated genes under TAC. A. Upregulated genes by panther Pathway category. B. Downregulated genes by Panther pathway category.

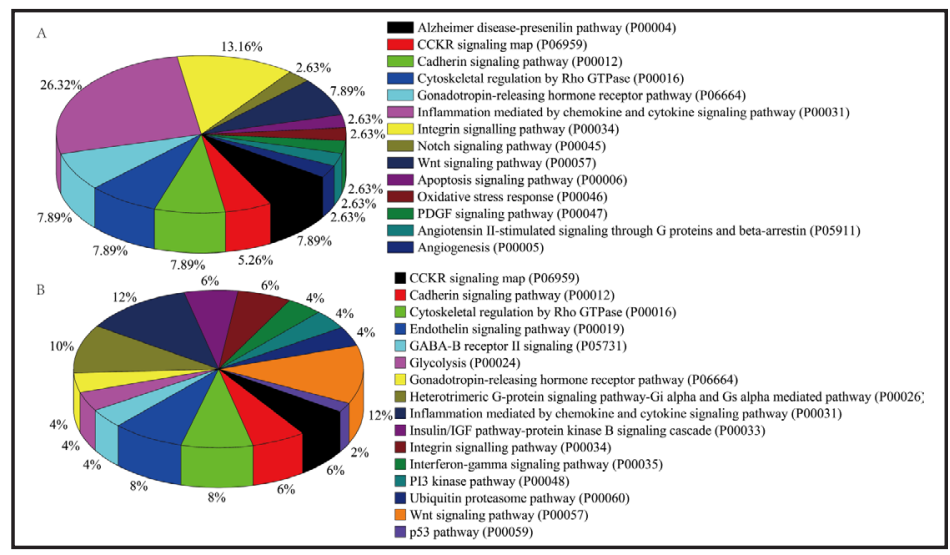


Fig. 4. Protein-protein interaction network of upand downregulated genes under TAC. Nodes are colored by regulation trend: upregulated (green) and downregulated (red). Edges are colored based on the number of connections: few connections (gray) and highly connected (blue). Node size is proportional to the $\mathrm{p}$ value: the lower the value, the larger the node.

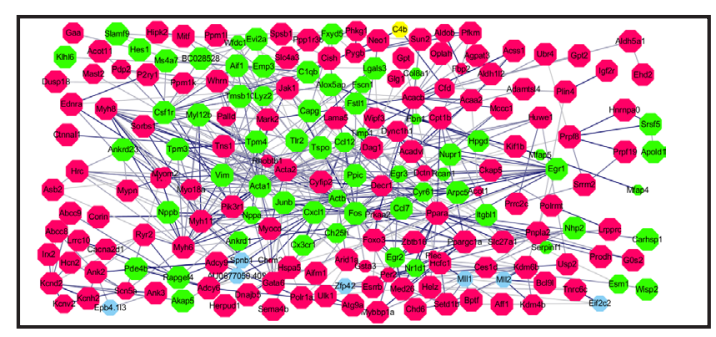

Table 1. KEGG pathway analysis of genes with the alteration of MeCP2

\begin{tabular}{|c|c|c|c|c|}
\hline $\begin{array}{l}\text { Alteration } \\
\text { trend }\end{array}$ & Pathway & count & $P$ value & genes \\
\hline \multirow{5}{*}{$\begin{array}{l}\text { The same } \\
\text { trend }\end{array}$} & mmu01200:Carbon metabolism & 23 & $\begin{array}{c}2.4 \times 10^{-} \\
7\end{array}$ & $\begin{array}{l}\text { DLST SHMT1 SHMT2 ACADM ACO2 ACADS ALDOB OGDHL PFKM DLAT GPT ACAT1 IDH3A } \\
\text { GOT2 PCX ACSS1 IDH3G H6PD SDHC ENO3 PCCB GPT2 PCCA }\end{array}$ \\
\hline & mmu04022:cGMP-PKG signaling pathway & 21 & 0.001 & $\begin{array}{l}\text { SLC8A1 CREB3 MRVI1 ADCY6 ATP1A1 ITPR2 VDAC1 PPIF EDNRRA RGS2 MYLK NFATC1 } \\
\text { ATP2A2 PDE2A ADCY9 GATA4 ADRA1A CACNA1C NFATC3 PIK3R1 INSR }\end{array}$ \\
\hline & mmu04020:Cakium signaling pathway & 18 & 0.01 & $\begin{array}{l}\text { PHKA2 SLC8A1 PHKG1 GRM1 ITPR2 VDAC1 PPIF EDNRA ATP2A2 ADCY9 CACNA1G PLCD3 } \\
\text { ADRA1A CACNA1H PRKACA CACNA1C CAMK2A MYLK }\end{array}$ \\
\hline & mmu04152:AMPK signaling pathway & 17 & 0.001 & $\begin{array}{l}\text { SREBF1 PPP2R1B CPT1B CREB3 PRKAG1 FOXO1 FOXO3 PFKM RPTOR TSC1 TSC2 RAB14 } \\
\text { ADRA1A SCD4 INSR LIPE PIK3R1 }\end{array}$ \\
\hline & $\begin{array}{l}\text { mmu05412: Arrhythmogenic right ventricular } \\
\text { cardiomyopathy (ARVC) }\end{array}$ & 9 & 0.03 & SGCG DSG2 DAG1 ITGA3 CTNNA1 CACNA1C TCF7L2 CTNNA3 SGCA \\
\hline \multirow{6}{*}{$\begin{array}{l}\text { The opposite } \\
\text { trend }\end{array}$} & mmu04068:FoxO signaling pathway & 16 & $\underset{4}{2.5 \times 10}$ & $\begin{array}{l}\text { PIK3CG SGK3 IGF1 PTEN G6PC3 CCNB1 NRAS TNFSF10 CCND1 KRAS CHUK MAPK12 } \\
\text { CCND2 SOS1 ARAF GADD45B }\end{array}$ \\
\hline & mmu04015:Rap1 signaling pathway & 20 & $\underset{4}{7.7 \times 10}$ & $\begin{array}{l}\text { ACTB PIK3CG ADCY7 GNAI2 IGF1 LPAR1 PRKCB CTNNB1 NRAS VEGFC PFN1 PFN2 KRAS } \\
\text { MAPK12 RAPGEF6 GNAS RAPGEF4 RAP1B CALM2 CSF1R }\end{array}$ \\
\hline & mmu04010:MAPK signaling pathway & 20 & 0.005 & $\begin{array}{l}\text { MEF2C PTPRR PPM1A PPM1B STK3 PRKCB NRAS FOS TNFRSF1A RPS6KA3 ATF4 DUSP3 } \\
\text { KRAS MAPK12 SOS1 RAP1B GADD45B CHUK HSPA8 DUSP6 }\end{array}$ \\
\hline & mmu04115: & 9 & 0.0 & CCNB1 CCND1 CCND2 IGF1 APAF1 CDK4 GADD45B PTEN \\
\hline & mmu04210:Apoptos & 7 & 0.03 & PIK3CG TNFRSF1A TNFSF10 BCL2 APAF1 CAPN2 CHUK \\
\hline & mmu04151:PI3K-Akt signaling pathway & 22 & 0.03 & $\begin{array}{l}\text { PIK3CG SGK3 PPP2R5C IGF1 LPAR1 CDK4 PTEN G6PC3 NRAS VEGFC ATF4 KRAS CCND1 } \\
\text { CCND2 SOS1 BCL2 GNG10 PIK3AP1 CHUK EIF4E2 PPP2R3C CSF1R }\end{array}$ \\
\hline
\end{tabular}

Lyz2 (lysozyme 2), Evi2a (ecotropic viral integration site 2a), Fbn1 (fibrillin 1), Junb (Jun B proto-oncogene), Mark2 (MAP/microtubule affinity regulating kinase 2), Decr1 (2, 4-dienoyl CoA reductase 1, mitochondrial), Alox5ap (arachidonate 5-lipoxygenase activating protein), Dcer1 (2, 4-dienoyl CoA reductase 1, mitochondrial), Egr1 (early growth response 1), Ttn (titin), Hrc (histidine rich calcium binding protein), Setd1b (SET domain containing 1B), Fos

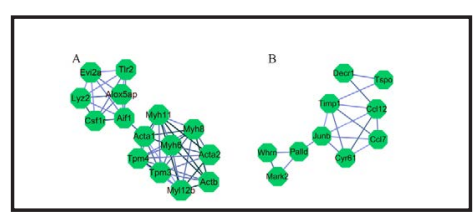

Fig. 5. Top 2 clusters from PPI networks under TAC. (FBJ osteosarcoma oncogene), Jak1 (Janus kinase 1), Foxo3 (forkhead box 03), and Ppara (peroxisome proliferator activated receptor alpha). These genes were mainly involved in angiogenesis, CCKR signaling and inflammation mediated by chemokine and cytokine signaling. The top 2 significant clusters analyzed by MCODE module analysis are shown in Fig. 5.

Genes that had the same and different regulation trends compared with MeCP2 under TAC and $r T A C$

In healthy mice, we compared the DEGs under TAC and rTAC and performed further alignment. MeCP2 was downregulated under TAC and upregulated under rTAC. According to the criterion of a p value $<0.01$, by selecting and aligning the DEGs from TAC and rTAC data, we screened 1055 genes that had the same expression-variation tendency as MeCP2 and 785 genes that had the opposite expression-variation tendency of MeCP2. GO-term analysis that that the DEGs' functions were mainly enriched in metabolic process, cardiovascular development, transcription and protein translation. Metabolic process includes glucose metabolic process, fatty acid catabolic process, amino acid metabolic process and energy metabolism. Cardiovascular development includes heart development, regulation of blood pressure, regulation of cardiac conduction, regulation of cardiac muscle cell contraction and regulation of epithelial cell proliferation. KEGG pathway analysis found that these genes were mainly enriched in metabolic pathway, signal transduction and apoptosis (Table 1).

Genes abnormally expressed with overexpressed MeCP2 under TAC

We screened 125 genes and performed GO term analysis of genes that were dysregulated when MeCP2 was overexpressed in mice under TAC. The GO-term BP analysis result was 


\section{Cellular Physiology Cell Physiol Biochem 2018;47:2380-2387 \begin{tabular}{l|l} 
DOI: 10.1159/000491610 & $\begin{array}{l}\text { O 2018 The Author(s). Published by S. Karger AG, Basel } \\
\text { www.karger.com/cpb }\end{array}$
\end{tabular} \\ Wang et al.: The Effect of MeCP2 on Heart Failure}

Fig. 6. GO-term BP analysis of abnormally expressed genes with MeCP2 overexpression under TAC. Nodes are colored based on the degree of enrichment: the highest density of enrichment (orange), the lowest (yellow).

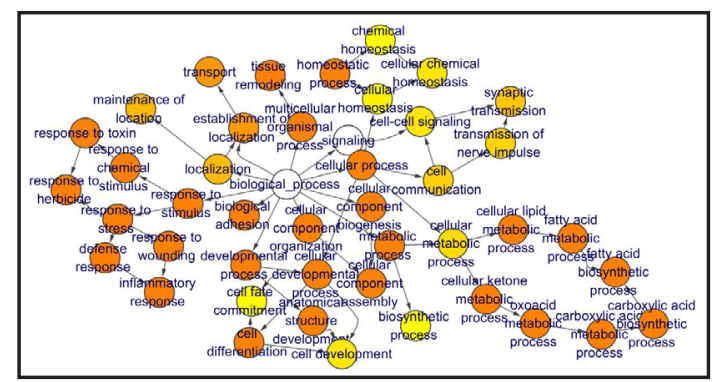

Fig. 7. Top 2 clusters from PPI networks. A. Module 1 with MeCP2 overexpression under TAC. B. Module 2 with MeCP2 overexpression under TAC. C. Module 1 with MeCP2 knockout under TAC. D. Module 2 with MeCP2 knockout under TAC.

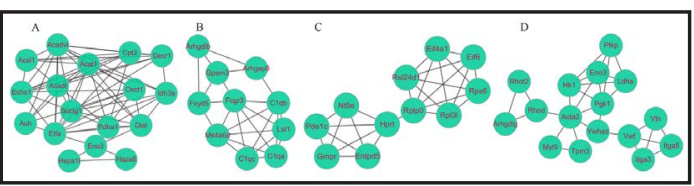

also enriched in metabolic process, regulation of cell migration, regulation of fibroblast proliferation and regulation of systemic arterial blood pressure by atrial natriuretic peptide (Fig. 6). Genes involved in heart development, such as CXADR, EDNRA, GAB1, ACADM, EP300, CRKL, and IRX4, were downregulated; genes involved in myocardial hypertrophy, such as HDAC2, TCAP, RYR2, and TTN, were downregulated; and many genes involved in mitochondrial activities (TSPO, DCN, COA3, NSUN4, MFN1, BAK1, CTSK, SOD2, OPA1), cation channel activity (PIRT, ANK3, KCNJ11), and heart trabecula formation (CAV3, RBP4, SRF) were dysregulated. MCODE module analysis is shown in Fig. 7A, B. The genes in modules A and $B$ were mainly involved in mitochondrial biological macromolecular synthesis, transport and metabolism, and the small GTPase-activating protein Rho mediated PDGF signaling pathway.

Genes abnormally expressed with ablation of MeCP2 under TAC

Sixty-six genes were screened. GO-term BP analysis showed the most density enrichment in biological regulation, cellular process, developmental process, metabolic process and immune system process. The most enriched pathways were adrenaline and noradrenaline biosynthesis, the Alzheimer disease-presenilin pathway, blood coagulation, insulin/IGF pathway-protein kinase B signaling cascade, nicotine degradation, oxidative stress response and TGF-beta signaling pathway. More than half of the DEGs that promote angiogenesis, such as ECM1, MTDH, PGF, and CYP1B1, were upregulated; genes involved in cardiac muscle hypertrophy, such as ECE1, AGT, and EDN1, were upregulated; genes involved in heart rate, such as SLC8A1, IRX5, DRD2, and POPDC2, were upregulated; genes involved in blood pressure, such as ACTA2, PPARG, MYH6, and NPR3 were dysregulated; and many genes involved in telomere maintenance (DCLRE1B, SP100, WRN, CTC1, ATM, TERF2), apoptosis (FOX01, GPX1, RARG, POR, JUN, STAT3), autophagy (BOK, BCL2, TRP53INP1, ATM), metabolic processes (ALAD, GPAT4, AUH), mitochondrial activities (POLG2, TK2, MTCH2, SNCA, TAZ, HIF1A, PPIF, BCL2, STAT3), inflammatory response (ABR, BCR, SOCS3, CD44, NT5E) and hormone transport (IGF2, POR, BLOC1S3, MREG) were dysregulated. The top 2 clusters analyzed by MCODE are shown in Fig. 7C, D. The top 2 modules were mainly enriched in mitochondrial nucleotide metabolism, energy metabolism, and inflammationmediated signaling pathways.

\section{Discussion}

MeCP2 is a pleiotropic DNA-binding protein that preferentially binds to methylated CpGs and regulates gene expression [17]. At first, much attention was focused on the role of MeCP2 in neurodevelopment because of the discovery of Rett syndrome caused by MeCP2 
mutations. Later, with the determination of the MeCP2 structure, it has become accepted that the effect of MeCP2 is likely to be modulated by the tissue- and cell-specific distribution of methylated and unmethylated CpG units, which clearly constitute an important control mechanism for gene expression [18]. MeCP2 is a multifunctional protein that mediates many downstream targets [19]. MeCP2 not only connects DNA methylation with histone deacetylation but also mediates two global epigenetic modifications, DNA methylation and histone methylation [20].

Cardiovascular events are a major cause of morbidity and mortality worldwide. Their occurrence and development are related to epigenetic regulation. MeCP2, as an epigenetic signature, plays a critical role in regulating cardiovascular diseases. Previous studies described the effect of MeCP2 on neuronal cells [21-23]. It was reported that MeCP2 induces a series of methylation events culminating in myofibroblast transdifferentiation and liver fibrogenesis [24]. It was found that MeCP2 overexpression results in downregulation of a crucial regulator for many endothelial cell functions, SIRT1, and contributes to the impaired angiogenic functions of endothelial cells [16]. Elevated MeCP2 expression has been reported to result in cardiac and skeletal abnormalities during development [25]. It was also reported that MeCP2 is upregulated in infarcted hearts and that inhibition of the MeCP2upstream microRNA miR-132 decreases myocardial contractility, reparative angiogenesis and interstitial fibrosis in infarcted hearts [26]. We analyzed the data from simulated rat heart failure caused by TAC and rTAC, which alter the expression of MeCP2, and screened many dysregulated genes under MeCP2 overexpression and knockout on a background of TAC. Our findings suggest that MeCP2 plays a key role in cardiovascular activities, including angiogenesis, heart development, blood pressure, myocardial contractility, cardiac conduction and myocardial hypertrophy and fibrosis.

Heart failure is a complicated clinical syndrome involving a multitude of genes and pathways. Our data show that MeCP2 plays an important role in the occurrence and development of heart failure and ventricular remodeling. We also found that MeCP2 is involved in the regulation of metabolic processes, including lipid metabolism, amino acid metabolism, nucleotide metabolism, glucose metabolism and energy metabolism. MeCP2 deficiency results in impaired mitochondrial function [27]. It was shown that MeCP2 regulates lipid homeostasis, and MeCP2 deletion in mice results in a host of severe metabolic defects caused by lipid accumulation, including insulin resistance, fatty liver, perturbed energy utilization, and adipose inflammation by macrophage infiltration [28]. It was also reported that a master regulator of metabolic homeostasis, insulin, is regulated by MeCP2 [29]. MeCP2 was also found to regulate genes involved in tumor processes. It was suggested that MeCP2 could upregulate c-Met expression by silencing miR-137 expression and thus contribute to the colorectal adenoma-carcinoma sequence and tumor progression [30]. Another study reported that MeCP2 promotes gastric cancer progression [31]. In this article, we found that MeCP2 participates in hormone modulation, for example, modulation of growth hormone $(\mathrm{GH})$, thyroid hormone and prolactin. It was reported that MeCP2 can modify the ghrelin/ GH/ IGF-1 axis in RTT [32].

In summary, MeCP2 participates in the somatic regulation of physiological and pathological activities. It plays a pivotal role not just in brain but in nearly all organs and tissues. It also plays a decisive role in heart failure, which is the end result of various cardiovascular diseases. We screened for genes aberrantly expressed in a simulated heart failure rat model and identified the genes that were abnormally changed with MeCP2 overexpression or knockout by using bioinformatic analysis. Further study of these genes may help to reveal the underlying molecular mechanisms of MeCP2 action in heart failure, leading to better therapies.

Our study has some limitations. The genes were selected from one GEO dataset and lack our own experimental verification. The data were from rats because of the difficulty of operating in humans, so our findings should be confirmed with human data. 


\section{Cellular Physiology Cell Physiol Biochem 2018;47:2380-2387 \begin{tabular}{l|l} 
DOI: 10.1159/000491610 & $\begin{array}{l}\text { O } 2018 \text { The Author(s). Published by S. Karger AG, Basel } \\
\text { www.karger.com/cpb }\end{array}$ \\
and Biochemistry
\end{tabular}}

Wang et al.: The Effect of MeCP2 on Heart Failure

\section{Acknowledgements}

This work was supported by The National Natural Science Foundation of China (No. 81471399).

\section{Disclosure Statement}

The authors declare tot have no competing interests.

\section{References}

1 Baker SA, Chen L, Wilkins AD, Yu P, Lichtarge O, Zoghbi HY: An AT-hook domain in MeCP2 determines the clinical course of Rett syndrome and related disorders. Cell 2013;152:984-996.

-2 Bird AP, Wolffe AP: Methylation-induced repression--belts, braces, and chromatin. Cell 1999;99:451-454.

-3 Baubec T, Ivanek R, Lienert F, Schubeler D: Methylation-dependent and -independent genomic targeting principles of the MBD protein family. Cell 2013;153:480-492.

4 Amir RE, Van den Veyver IB, Wan M, Tran CQ, Francke U, Zoghbi HY: Rett syndrome is caused by mutations in X-linked MECP2, encoding methyl-CpG-binding protein 2. Nat Genet 1999;23:185-188.

5 Ben-Shachar S, Chahrour M, Thaller C, Shaw CA, Zoghbi HY: Mouse models of MeCP2 disorders share gene expression changes in the cerebellum and hypothalamus. Hum Mol Genet 2009;18:2431-2442.

6 Chahrour M, Jung SY, Shaw C, Zhou X, Wong ST, Qin J, Zoghbi HY: MeCP2, a key contributor to neurological disease, activates and represses transcription. Science 2008;320:1224-1229.

7 Samaco RC, Mandel-Brehm C, McGraw CM, Shaw CA, McGill BE, Zoghbi HY: Crh and Oprm1 mediate anxiety-related behavior and social approach in a mouse model of MECP2 duplication syndrome. Nat Genet 2012;44:206-211.

-8 Skene PJ, Illingworth RS, Webb S, Kerr AR, James KD, Turner DJ, Andrews R, Bird AP: Neuronal MeCP2 is expressed at near histone-octamer levels and globally alters the chromatin state. Mol Cell 2010;37:457468.

9 Adachi M, Keefer EW, Jones FS: A segment of the Mecp2 promoter is sufficient to drive expression in neurons. Hum Mol Genet 2005;14:3709-3722.

10 Coy JF, Sedlacek Z, Bachner D, Delius H, Poustka A: A complex pattern of evolutionary conservation and alternative polyadenylation within the long 3"-untranslated region of the methyl-CpG-binding protein 2 gene (MeCP2) suggests a regulatory role in gene expression. Hum Mol Genet 1999;8:1253-1262.

11 Reichwald K, Thiesen J, Wiehe T, Weitzel J, Poustka WA, Rosenthal A, Platzer M, Stratling WH, Kioschis P: Comparative sequence analysis of the MECP2-locus in human and mouse reveals new transcribed regions. Mamm Genome 2000;11:182-190.

12 Shahbazian MD, Antalffy B, Armstrong DL, Zoghbi HY: Insight into Rett syndrome: MeCP2 levels display tissue- and cell-specific differences and correlate with neuronal maturation. Hum Mol Genet 2002;11:115124.

13 Hong Y, Cao H, Wang Q Ye J, Sui L, Feng J, Cai X, Song H, Zhang X, Chen X: MiR-22 may Suppress Fibrogenesis by Targeting TGFbetaR I in Cardiac Fibroblasts. Cell Physiol Biochem 2016;40:1345-1353.

14 Tao H, Yang JJ, Hu W, Shi KH, Deng ZY, Li J: MeCP2 regulation of cardiac fibroblast proliferation and fibrosis by down-regulation of DUSP5. Int J Biol Macromol 2016;82:68-75.

15 Mayer SC, Gilsbach R, Preissl S, Monroy Ordonez EB, Schnick T, Beetz N, Lother A, Rommel C, Ihle H, Bugger H, Ruhle F, Schrepper A, Schwarzer M, Heilmann C, Bonisch U, Gupta SK, Wilpert J, Kretz O, von Elverfeldt D, Orth J, Aktories K, Beyersdorf F, Bode C, Stiller B, Kruger M, Thum T, Doenst T, Stoll M, Hein L: Adrenergic Repression of the Epigenetic Reader MeCP2 Facilitates Cardiac Adaptation in Chronic Heart Failure. Circ Res 2015;117:622-633.

16 Volkmann I, Kumarswamy R, Pfaff N, Fiedler J, Dangwal S, Holzmann A, Batkai S, Geffers R, Lother A, Hein L, Thum T: MicroRNA-mediated epigenetic silencing of sirtuin1 contributes to impaired angiogenic responses. Circ Res 2013;113:997-1003. 


\section{Cellular Physiology Cell Physiol Biochem 2018;47:2380-2387 \begin{tabular}{l|l} 
DOI: 10.1159/000491610 & $\begin{array}{l}\text { O 2018 The Author(s). Published by S. Karger AG, Basel } \\
\text { www.karger.com/cpb }\end{array}$
\end{tabular} \\ Wang et al.: The Effect of MeCP2 on Heart Failure}

17 Yang T, Ramocki MB, Neul JL, Lu W, Roberts L, Knight J, Ward CS, Zoghbi HY, Kheradmand F, Corry DB: Overexpression of methyl-CpG binding protein 2 impairs T(H)1 responses. Sci Transl Med 2012;4:163ra158.

18 Hansen JC, Ghosh RP, Woodcock CL: Binding of the Rett syndrome protein, MeCP2, to methylated and unmethylated DNA and chromatin. IUBMB Life 2010;62:732-738.

19 Hite KC, Adams VH, Hansen JC: Recent advances in MeCP2 structure and function. Biochem Cell Biol 2009;87:219-227.

-20 Fuks F, Hurd PJ, Wolf D, Nan X, Bird AP, Kouzarides T: The methyl-CpG-binding protein MeCP2 links DNA methylation to histone methylation. J Biol Chem 2003;278:4035-4040.

-21 Chen RZ, Akbarian S, Tudor M, Jaenisch R: Deficiency of methyl-CpG binding protein-2 in CNS neurons results in a Rett-like phenotype in mice. Nat Genet 2001;27:327-331.

22 Chen WG, Chang Q, Lin Y, Meissner A, West AE, Griffith EC, Jaenisch R, Greenberg ME: Derepression of BDNF transcription involves calcium-dependent phosphorylation of MeCP2 Science 2003;302:885-889.

23 Guy J, Hendrich B, Holmes M, Martin JE, Bird A: A mouse Mecp2-null mutation causes neurological symptoms that mimic Rett syndrome. Nat Genet 2001;27:322-326.

24 Mann J, Chu DC, Maxwell A, Oakley F, Zhu NL, Tsukamoto H, Mann DA: MeCP2 controls an epigenetic pathway that promotes myofibroblast transdifferentiation and fibrosis. Gastroenterology 2010;138:705714, 714 e701-704.

-25 Alvarez-Saavedra M, Carrasco L, Sura-Trueba S, Demarchi Aiello V, Walz K, Neto JX, Young JI: Elevated expression of MeCP2 in cardiac and skeletal tissues is detrimental for normal development. Hum Mol Genet 2010;19:2177-2190.

-26 Katare R, Riu F, Mitchell K, Gubernator M, Campagnolo P, Cui Y, Fortunato O, Avolio E, Cesselli D, Beltrami AP, Angelini G, Emanueli C, Madeddu P: Transplantation of human pericyte progenitor cells improves the repair of infarcted heart through activation of an angiogenic program involving micro-RNA-132 Circ Res 2011;109:894-906.

-27 Park MJ, Aja S, Li Q, Degano AL, Penati J, Zhuo J, Roe CR, Ronnett GV: Anaplerotic triheptanoin diet enhances mitochondrial substrate use to remodel the metabolome and improve lifespan, motor function, and sociability in MeCP2-null mice. PLoS One 2014;9:e109527.

28 Kyle SM, Saha PK, Brown HM, Chan LC, Justice MJ: MeCP2 co-ordinates liver lipid metabolism with the NCoR1/HDAC3 corepressor complex. Hum Mol Genet 2016;25:3029-3041.

29 Kuroda A, Rauch TA, Todorov I, Ku HT, Al-Abdullah IH, Kandeel F, Mullen Y, Pfeifer GP, Ferreri K: Insulin gene expression is regulated by DNA methylation. PLoS One 2009;4:e6953.

-30 Chen T, Cai SL, Li J, Qi ZP, Li XQ, Ye LC, Xie XF, Hou YY, Yao LQ, Xu MD, Zhou PH, Xu JM, Zhong YS: Mecp2mediated Epigenetic Silencing of miR-137 Contributes to Colorectal Adenoma-Carcinoma Sequence and Tumor Progression via Relieving the Suppression of c-Met. Sci Rep 2017;7:44543.

31 Zhao L, Liu Y, Tong D, Qin Y, Yang J, Xue M, Du N, Liu L, Guo B, Hou N, Han J, Liu S, Liu N, Zhao X, Wang L, Chen Y, Huang C: MeCP2 Promotes Gastric Cancer Progression Through Regulating FOXF1/Wnt5a/betaCatenin and MYOD1/Caspase-3 Signaling Pathways. EBioMedicine 2017;16:87-100.

-32 Hara M, Nishi Y, Yamashita Y, Hirata R, Takahashi S, Nagamitsu S, Hosoda H, Kangawa K, Kojima M, Matsuishi T: Relation between circulating levels of GH, IGF-1, ghrelin and somatic growth in Rett syndrome. Brain Dev 2014;36:794-800. 